\title{
Oviposition Preference for Young Plants by the Large Cabbage Butterfly (Pieris brassicae) Does not Strongly Correlate with Caterpillar Performance
}

\author{
Minghui Fei $^{1} \cdot$ Jeffrey A. Harvey $^{1,2} \cdot$ Yi Yin $^{1} \cdot$ Rieta Gols $^{3}$ (D)
}

Received: 9 March 2017 /Revised: 8 May 2017 / Accepted: 28 May 2017 / Published online: 15 June 2017

(C) The Author(s) 2017. This article is an open access publication

\begin{abstract}
The effects of temporal variation in the quality of short-lived annual plants on oviposition preference and larval performance of insect herbivores has thus far received little attention. This study examines the effects of plant age on female oviposition preference and offspring performance in the large cabbage white butterfly Pieris brassicae. Adult female butterflies lay variable clusters of eggs on the underside of short-lived annual species in the family Brassicaceae, including the short-lived annuals Brassica nigra and Sinapis arvensis, which are important food plants for P. brassicae in The Netherlands. Here, we compared oviposition preference and larval performance of $P$. brassicae on three age classes (young, mature, and pre-senescing) of B. nigra and S. arvensis plants. Oviposition preference of $P$. brassicae declined with plant age in both plant species. Whereas larvae performed similarly on all three age classes in B. nigra, preference and performance were weakly correlated in S. arvensis. Analysis of primary (sugars and amino acids) and secondary (glucosinolates) chemistry in the plant shoots revealed that differences in their quality and quantity were more pronounced with respect to tissue type (leaves vs. flowers) than among different developmental stages of both plant species.
\end{abstract}

Electronic supplementary material The online version of this article (doi:10.1007/s10886-017-0853-9) contains supplementary material, which is available to authorized users.

Rieta Gols

Rieta.gols@wur.nl

1 Department of Terrestrial Ecology, Netherlands Institute of Ecology, Wageningen, The Netherlands

2 Department of Ecological Sciences, Section Animal Ecology, VU University Amsterdam, Amsterdam, The Netherlands

3 Laboratory of Entomology, Wageningen University and Research, Wageningen, The Netherlands
Butterflies of $P$. brassicae may prefer younger and smaller plants for oviposition anticipating that future plant growth and size is optimally synchronized with the final larval instar, which contributes $>80 \%$ of larval growth before pupation.

Keywords Brassicaceae - Butterfly oviposition choice · Glucosinolates · Insect herbivores · Insect performance $\cdot$ Plant development $\cdot$ Plant ontogeny $\cdot$ Primary metabolites .

Secondary metabolites

\section{Introduction}

The suitability of a food plant for growth and development of herbivorous insects is generally determined by a combination of sufficiently high concentrations of primary metabolites and low concentrations of secondary metabolites (Scriber and Slansky 1981). Primary metabolites, such as proteins, especially carbohydrates, and fatty acids are essential for the growth, development, and reproduction of all living organisms (Schoonhoven et al. 2005). Given its central role in most metabolic processes, nitrogen is considered to be of critical importance for the growth and development of insects and is often limiting in plant tissues reducing the quality of plants as food (Lawton and McNeill 1979; Mattson 1980; Scriber and Slansky 1981). Secondary metabolites often play a defensive role in resistance against insect herbivores (Fraenkel 1959; Iason et al. 2012; Mithöfer and Boland 2012; Schoonhoven et al. 2005). Resistance may involve traits that deter oviposition or feeding and interfere with the insect's physiology once tissues have been ingested (Schoonhoven et al. 2005). However, most insect herbivores are dietary specialists and thus feed on only a few species or closely related plants that produce phylogenetically conserved secondary metabolites (Loxdale et al. 2011; Schoonhoven et al. 2005). 
Consequently, many insect herbivores have evolved various adaptations to deal with adverse phytochemicals (Despres et al. 2007; Nishida 2002; Winde and Wittstock 2011) and many species even use certain phytochemicals for host plant recognition (Renwick 1989).

The production of primary and secondary metabolites is largely genetically controlled and often varies with plant age and in response to biotic and abiotic factors such as rainfall, temperature and insect feeding and pathogen attack (Barton and Koricheva 2010; Karban and Baldwin 1997; Schoonhoven et al. 2005). Nitrogen demands (and N levels) are often higher in young growing tissues of plants and decline with age (Mattson 1980). Concentrations of secondary metabolites in plant tissues are also dynamic and can change with time. Using meta-analysis, Barton and Koricheva (2010) reviewed ontogenetic changes in plant secondary metabolites across a range of plant taxa and found that constitutive concentrations of all types of secondary metabolites significantly increased from the seedling to the adult stage in both herbaceous and woody plants.

Adult females of insect herbivores must find suitable food plants for their offspring, and these plants are often found in habitats that vary in structural and chemical complexity. When the temporal availability and quality of suitable food plants is highly predictable, searching for oviposition sites is often closely synchronized with these parameters (Wolda 1988). For example, larval feeding stages of monophagous insects that have only one generation per year (i.e., are univoltine) usually occur only when suitable plant tissues, such as young leaves, are available (van Asch et al. 2010). In contrast, when adult females of multivoltine herbivorous insects that feed on short-lived herbaceous plant species are searching for oviposition sites, they are faced with a range of challenges. For example, different generations have to find suitable food plant species that may differ in quality due to differences in plant age, which can potentially have significant effects on the development, survival and fitness of their progeny.

According to the preference-performance hypothesis, females should maximize their fitness by laying eggs on plants on which the offspring perform the best (Jaenike 1990; Mayhew 1998). However, this hypothesis ignores the fact that the relationship between female preference and offspring performance may also be influenced by other ecological factors such as predation risk, the dietary breadth of the herbivores and food plant availability at the time of oviposition (Gripenberg et al. 2010). These other considerations may explain why the evidence supporting the preferenceperformance hypothesis is variable (Mayhew 2001; Scheirs et al. 2005). When the quality of host plants varies unpredictably over time, females may fail to evolve the ability to choose the plant type that would be most suitable for development of their offspring (Cronin et al. 2001; Gripenberg et al. 2010). Multivoltine insect herbivores may be forced to use different host plant species over the course of a growing season, depending on what is available at a specific time and their performance may be constrained by temporal and speciesspecific variation in host plant quality as well as quantity (in the case of gregarious species) (Fei et al. 2014; Fei et al. 2016a; Gols et al. 2007).

In this study, we examine female oviposition preference and offspring performance of the large cabbage white butterfly Pieris brassicae L. (Lepidoptera: Pieridae) in relation to plant ontogeny. Larval stages of this herbivore feed only on plants that contain glucosinolates, defensive secondary metabolites characteristic of species in the plant family Brassicaceae (Fahey et al. 2001). Adult females and larvae of P. brassicae use glucosinolates as oviposition and feeding stimulants, respectively (Renwick 2002; van Loon et al. 1992). Most of the host plant species of $P$. brassicae are short-lived annuals that are present in the field for only two to three months (Feltwell 1982). These include cabbage crops and mustard oil species on which P. brassicae is considered an important pest species across much of Eurasia (Feltwell 1982). In the Netherlands, $P$. brassicae has two to three generations per year. In relation to the phenology of the herbivore and its potential host plants, amongst others, the charlock mustard Sinapis arvensis L., which grows in late spring and early summer and the black mustard, Brassica nigra L., which grows in middle to late summer, are considered important food plants for this herbivore. These ephemeral plant species often grow in dense populations, which is important because $P$. brassicae lays eggs in clutches of 30-100 eggs on a single plant and at least several plants are required to support the complete development of an entire brood (Fei et al. 2016a). Furthermore, previous work has shown that $P$. brassicae caterpillars not only feed on the leaves, but that older larvae move up the plants and feed on the flowers (Smallegange et al. 2007). When caterpillars were only allowed to consume flowers, they developed faster and obtained a higher final caterpillar mass compared to conspecifics that were restricted to feed on leaves (Smallegange et al. 2007). These results suggest that the flowers are of higher nutritional quality than the leaves.

In the field, when female $P$. brassicae butterflies are searching for oviposition sites, they often encounter plant individuals of variable age and potentially quality. These plants may also vary considerably in size, as $B$. nigra plants grow as high as $2 \mathrm{~m}$ or more, depending on soil quality, access to light, competition with other plants and nutrient availability. The aims of this study are to 1) investigate whether female butterflies distinguish between different developmental stages of the two host plant species for oviposition and 2) to determine whether female butterflies lay their eggs on plants in the developmental stage that is best for growth and development of their offspring. Nutritional quality and levels of defensive chemistry are usually higher in young developing leaves than in old mature leaves (Bowers and Stamp 1993; Scriber and 
Slansky 1981). Also, glucosinolate concentrations are generally higher in newly developed leaves than in older leaves (Gols et al. 2007). As $P$. brassicae caterpillars are specialist herbivores on several species of brassicaceous plants including the two species studied here, we predict that larval development will not be affected by variable concentrations of glucosinolates. Instead, larval performance will be better on younger plants that are of higher nutritional quality in terms of primary metabolites than on older plants. To link larval performance with qualitative characteristics of the plant tissues, we analyzed and quantified primary (amino acids and sugars) and secondary metabolites (glucosinolates) in tissues collected from plants in different developmental stages and correlated these with larval performance variables using comprehensive multivariate statistics.

\section{Methods and Materials}

Plants and Insects Sinapis arvensis and Brassica nigra grow in disturbed sites, such as along river flood plains and in areas where soil is disturbed and nutrient availability is high. In the Netherlands, these two plant species are very important food plants for $P$. brassicae. Seeds of both species were collected from several plants $(>15)$ in wild populations growing naturally near Wageningen, the Netherlands. Seeds were germinated and seedlings were subsequently transferred to $1.1-\mathrm{L}$ pots filled with peat soil ('Lentse potgrond' no.4; lent, The Netherlands). Plants were grown in a greenhouse at $21 \pm 2{ }^{\circ} \mathrm{C}$ (day) and $16 \pm 2{ }^{\circ} \mathrm{C}$ (night), $50 \%$ r.h., and a photoperiod of at least $16 \mathrm{hr}$. If the light dropped below $225 \mu \mathrm{mol}$ photons $\mathrm{m}^{2} \mathrm{~s}^{-1}$ during the $16-\mathrm{h}$ photoperiod, supplementary illumination was applied by sodium lamps. The plants were watered twice a week during the first 3 weeks of development and watering was then gradually increased to once daily. When the plants were 3 weeks old, they were fertilized once a week with Hoagland solution which was applied to the soil. Watering and fertilization continued during the experiments. This fertilization regime is necessary to sustain normal growth of these fast growing plants in small pots.

To investigate the effect of plant developmental stage (and size) on insect preference and performance, plants of different age classes were reared by sowing the seeds and growing the plants at different time points. Because $S$. arvensis has a shorter life cycle than $B$. nigra, different age classes were used for the two species. Seeds of $S$. arvensis were germinated at two-week intervals and plants used in the experiment were 3 , 5 and 7 weeks old, respectively. In the greenhouse, three-week old $S$. arvensis plants are in the transition between the vegetative and reproductive developmental phase; plants are still developing leaves, but flower buds are starting to develop as well. Five-week old $S$. arvensis plants are flowering and the leaves are still succulent (mid-reproductive phase), whereas seven-week old plants are still flowering, but the leaves are starting to senescence (late-reproductive phase). Seeds of $B$. nigra were germinated at three-week intervals and plants used in the experiments were 3, 6 and 9 weeks old, respectively. Three-week old $B$. nigra plants are still in the vegetative developmental phase, six-week old plants are in the late vegetative/early reproductive phase, whereas nine-week old plants are fully flowering and still have green leaves (=midreproductive phase).

Cultures of $P$. brassicae were maintained in a climate room at $22 \pm 2{ }^{\circ} \mathrm{C}, 50-70 \%$ r.h. and a photoperiod of at least $16 \mathrm{~h}$. $P$. brassicae which had been collected from the field in the previous summer were reared on Brussels sprout plants (B. oleracea var. gemmifera, cv. Cyrus) for approximately 10 generations at the Laboratory of Entomology, Wageningen University.

Experiment 1. Butterfly Oviposition Preference in Relation to Plant Ontogenetic Stage Larvae of $P$. brassicae were reared on Brussels sprouts and pupae were collected from the rearing cages and transferred to a clean cage $(60 \times 60 \times 180 \mathrm{~cm})$ placed in the experimental greenhouse Newly emerged adult butterflies were provided with a $(20 \%)$ honey solution applied to cotton wool in blue plastic caps to promote feeding activity. The butterflies were 3-5 days old when used in the choice bioassays.

Oviposition preference was determined in three-choiceexperiments in tents $(2 \times 2 \times 2 \mathrm{~m})$ placed outside in the garden of the Netherlands Institute of Ecology on bare soil. Plants of the three different age classes, one plant from each class, were prepared as described in the Plants and insects section. Bioassays were performed for each plant species separately.

Single plants from the three different age classes were randomly placed in one of three corners of the tent. Unmated females only lay a few infertile eggs. As not all females in the rearing cage may have mated, one female and one male butterfly were released in the tent to allow additional mating. A bioassay was terminated when a female butterfly had laid her first egg clutch which was checked every hour. The age class of the plant on which the eggs were laid was recorded. The bioassay was repeated at least 50 times for each plant species. Bioassays were conducted from June to August. New plants and butterflies were used for each replicate.

Plants of the three age classes differed in size. To determine whether differences in size of the plants influenced oviposition decisions, we also measured total leaf surface area and plant height of at least ten plants per age class. For leaf surface area measurements, all the leaves from single plants were collected and scanned using a photo scanner (Perfection4990; Epson, Japan). The leaf surface area was determined using the software WinFOLIA (Regent Instruments, Sainte-Foy, Canada). 
Experiment 2a. Ontogenetic Effects of Plant Quality on Herbivore Performance To obtain larvae for the performance bioassay, plants of the various developmental stages were placed in the rearing cage with $P$. brassicae butterflies. The females used for oviposition here were not the same as used in the oviposition bioassays. Since $P$. brassicae eggs need about one week to hatch under the greenhouse conditions used in this study, B. nigra and $S$. arvensis plants were prepared one week before they were used in the experiments. Female butterflies were allowed to oviposit on these plants for $24 \mathrm{~h}$. The following day, the plants were removed from the butterfly cage and transferred to the experimental greenhouse. Larvae hatching from the eggs were allowed to feed upon their natal plants until they reached the second instar. Egg hatching was close to $100 \%$ and mortality of first instar caterpillars was very low $(<5 \%)$. Using a fine paint brush, five cohorts (= replicates) of 20 randomly selected 1-day old L2 larvae were transferred to cages containing four plants that were in the same developmental stage as the one on which the caterpillars had hatched and had been feeding previously. As young P. brassicae caterpillars prefer to feed gregariously, they were introduced onto a single fully developed leaf of one plant in a cage.

Depending on the developmental stage of the plants, we used differently sized cages $(40 \times 40 \times 60 \mathrm{~cm}$ and $60 \times 60 \times 180$, respectively, Vermandel, Hulst, The Netherlands). In order to exclude a possible effect of temperature differences in the two differently-sized cages, we measured the temperature in the middle of the cages twice a day. There was no significant difference in temperatures between the two cage sizes. Caterpillars were allowed to move and feed freely on the plants within a cage until they pupated. Pupation was recorded daily and pupae were collected and weighed on an analytical balance ( $0.1 \mathrm{mg}$ accuracy). During the experiment, extra plants of the same age as the plants in the cage were added when needed (that is when more than $50 \%$ of the plant tissues had been consumed). As proxies for insect performance, we measured insect development time from egg hatching to pupation, pupal fresh mass and survival to pupation. The conditions were the same as for plant growth.

\section{Experiment 2b. Plant Ontogenetic Changes in Chemical} Profiles During the larval performance bioassays, plant tissues were collected for analysis of primary (sugars and amino acids) and secondary metabolites (glucosinolates) from both $B$. nigra and $S$. arvensis plants in the three age classes. Tissues were sampled from the plants when the caterpillars had been feeding on them for 9 days. Caterpillars were removed before sampling. Leaf tissues were collected by excising five $7 \mathrm{~mm}$ (diameter) discs from each of four fully developed green leaves of two plants within each cage. As P. brassicae caterpillars feed on both leaves and flowers (Smallegange et al. 2007), we also collected flowers for chemical analysis. Eight stems with flowers were cut off the same plants from which leaf tissues had been collected. In addition, leaf and flower tissues were sampled from control plants in the same three age classes that had not been exposed to herbivory, but were otherwise treated the same as those exposed to caterpillar feeding. Tissues were sampled in the morning between 10:00 and 12:00. Leaf discs and flowers, respectively, were pooled per cage in tinfoil and frozen in liquid nitrogen immediately after sampling and stored at $-80{ }^{\circ} \mathrm{C}$ until further processing. Samples were freeze-dried and pulverized with a grinding machine (Retsch GmbH, type MM 301).

For quantification and identification of soluble sugars, amino acids, and glucosinolates, one global extraction was conducted for each sample. Approximately $50.0 \mathrm{mg}$ of finely ground plant material was suspended in $1.0 \mathrm{ml} 70 \% \mathrm{MeOH}$ in water (vol/vol), vortexed, and immediately boiled for $5 \mathrm{~min}$ to inactive enzymes. Eppendorf tubes containing the sample were incubated in an ultrasonic bath for $15 \mathrm{~min}$ and then centrifuged for $10 \mathrm{~min}$ at $10000 \mathrm{~g}$. The extraction was repeated with the pellet, omitting the boiling step. Both supernatants were combined per sample and supplemented with $70 \%$ $\mathrm{MeOH}$ to a final volume of $2.0 \mathrm{ml}$. This 'stock' extract was stored at $-20{ }^{\circ} \mathrm{C}$ until further analysis.

Soluble sugars were separated by ion-exchange high-performance liquid chromatography (HPLC) with a CarboPac PA1 main column $(2 \times 250 \mathrm{~mm})$ and a CarboPac PA1 guard column $(2 \times 50 \mathrm{~mm})$. Sugars were separated using an isocratic gradient mixture of $10 \% 1 \mathrm{M} \mathrm{NaOH}$ in water at a flow rate of $0.25 \mathrm{ml} \mathrm{min}{ }^{-1}$ and a column temperature of $20{ }^{\circ} \mathrm{C}$ (for more details see van Dam and Oomen (2008)). A reference solution containing $54.9 \mu \mathrm{M}$ sorbitol and mannitol, 29.2 $\mu \mathrm{M}$ trehalose, sucrose and melibiose, and $55.5 \mu \mathrm{M}$ glucose and fructose was diluted to obtain calibration standards of $2.5,5$ and $7.5 \mathrm{ppm}$, respectively, for construction of a reference curve. Quantification was based on these reference curves.

Amino acids were analyzed on an Ion-exchange HPLC with an AminoPac PA10 main column $(2 \times 250 \mathrm{~mm})$ and an AminoPac PA10 guard column $(2 \times 50 \mathrm{~mm})$ and were separated with a tenary gradient. The method is described in detail in van Dam and Oomen (2008). For quantification and identification a reference sample was prepared containing the Sigma AA-S-18 amino acid standard (Sigma, St Louis, MO, USA) supplemented with asparagine, glutamine and tryptophan each at a concentration of $2.5 \mathrm{mM} \mathrm{ml}^{-1}$.

For glucosinolate analysis, $1 \mathrm{ml}$ of the stock was applied to a DEAE-Sephadex A25 column and desulfated with sulfatase (type H-1 from Helix pomatia, Sigma-Aldrich). Glucosinolates were separated by HPLC with an acetonitrile-water gradient $(2-65 \%$ acetonitrile from 0 to $30 \mathrm{~min}$; flow $0.75 \mathrm{ml} \mathrm{min}{ }^{-1}$; column temperature; $40^{\circ} \mathrm{C}$ ) on a reversed phase Alltima C-18 column $(150 \times 4.6 \mathrm{~mm})$. For detection of the glucosinolates a photodiode array detector was used set at $229 \mathrm{~nm}$ as the integration wavelength. The 
method is described in detail in van van Dam et al. (2004). For quantification, sinigrin was used as an external standard. Glucosinolate identification was based on their retention times and their UV spectra were compared to those of pure compounds provided by M. Reichelt (Max Planck Institute for Chemical Ecology, Jena, Germany) and a certified rapeseed standard (community Bureaus of Reference, Brussels, Belgium, code BCR-367 R).

Statistical Analyses Data were analyzed for the two plant species separately. To analyze butterfly oviposition preference, we used $\chi^{2-}$ tests comparing the observed oviposition counts on the three age classes with an expected distribution of $1: 1: 1$. When the test result was significant, we conducted pairwise $\chi^{2-}$ tests with $\alpha=0.05 / 3$ to correct for type I errors (Bonferroni correction). Data on plant height and leaf area were analyzed using one-way ANOVA with plant developmental class as a fixed factor, followed by Tukey-Kramer multiple pair-wise comparison tests to reveal differences between means.

In the experiments measuring larval performance on plants belonging to different age classes, the five cages, each containing 20 caterpillars, served as experimental units. Therefore, we first calculated the mean values per cage for the two performance variables, pupal mass and egg-to-pupa development time. These mean values were then subjected to one-way ANOVA with plant developmental class as a fixed factor. Tukey-Kramer multiple comparison tests between means were conducted when the ANOVA models were significant. The ANOVA tests were carried out using SPSS (IBM SPSS, statistics version 19).

We used a multivariate statistical approach, i.e. constrained principal component redundancy analysis (RDA), to determine differences in plant chemistry among the three age classes, the two tissue types (leaves and flowers) and damage status (control or damaged by P. brassicae feeding). The data were 'constrained' by classifying them according to tissue type, developmental stage and damage status. Multivariate partitioning of variance analysis was used to determine how much of the variation in the chemistry variables was uniquely explained by each of the three factors. The multivariate counterpart of the ordinary F-ratio in univariate statistics was calculated using the sums of squares totaled across all response variables to yield the $\mathrm{H}_{0} \mathrm{~F}$-statistic, which is referred to as a pseudo F-statistic. Monte Carlo permutation (default setting of 999 permutations) tests were used to determine whether model terms (tissue type, developmental stage, damage status) were significant or not (ter Braak and Šmilauer 2012). RDA was also used to analyze whether there is a significant correlation between compounds and herbivore fitness parameters (biomass and development time). For analysis of the chemical data we used the concentrations of the various sugars, amino acids, and glucosinolates, expressed as amount of chemical per unit dry weight of tissue and for the herbivore performance data we used the corresponding mean pupal weight and developmental time. Data (log-transformed and mean-centered) were analyzed for the two plant species separately. RDA analysis on all chemical data was performed in Canoco version 5.03 (ter Braak and Šmilauer, The Netherlands).

\section{Results}

Experiment 1: Butterfly Oviposition Preference in Relation to Plant Ontogenetic Stage All eggs were laid on the leaves of the plant and not on the flowers when they were present. Female $P$. brassicae butterflies clearly discriminated between the three age classes of $S$. arvensis $\left(\chi_{2}^{2}=19.7\right.$, $P<0.001)$ and B. nigra $\left(\chi_{2}^{2}=22.8, P<0.001\right)$, respectively, when deciding where to oviposit (Fig. 1). Oviposition preference order declined with plant age for both plant species (Fig. 1). Females preferred to lay eggs on plants in the vegetative or early reproductive developmental phase over plants that were in the middle to late reproductive phase.

Plant height differed among the three age classes in both plant species (S. arvensis: $\mathrm{F}_{2,35}=147, P<0.001$, Fig. 2a; B. nigra: $F_{2,44}=101, P<0.001$; Fig. $2 \mathrm{c}$ ) and correlated positively with plant age. In addition, there was an effect of age class on total leaf surface area $\left(S\right.$. arvensis, $F_{2,77}=17.7$, $P<0.001$, Fig. $2 b ; B$. nigra, $F_{2,57}=21.7, P<0.001$, Fig. 2d). In $S$. arvensis, total leaf surface area was higher in the middle reproductive phase than in plants that were in the vegetative/early reproductive phase (leaves on these plants were still expanding) and late reproductive phase (leaves on these plants started to wilt and die) (Fig. 2b). In B. nigra, vegetative plants were still developing new leaves and total leaf area was lowest in these plants. Total leaf area was intermediate in plants in the mid-reproductive phase on which the older leaves started to wilt and die, and leaf area was highest in plants in the vegetative/early reproductive phase (Fig. 2d).

Experiment 2a: Ontogenetic Effects of Plant Quality on Herbivore Performance Both egg-to-pupa development time $\left(F_{2,14}=11.7, P=0.002\right)$ and pupal mass $\left(F_{2,14}=5.07\right.$, $P=0.026)$ were affected by the age class of the $S$. arvensis plants on which the insects had been reared. Insects developed slower and developed into smaller pupae with increasing age of the plants (Fig. 3a and c).

The effect of plant developmental stage on food plant quality for P. brassicae was less pronounced in $B$. nigra than in S. arvensis, and affected only pupal mass $\left(F_{2,14}=18.3\right.$, $P<0.001$, Fig. $3 b)$ and not egg-to-pupa development time $\left(F_{2,14}=2.09, P=0.17\right.$ Fig. $\left.3 \mathrm{~d}\right)$. Pupae were heavier on plants in both the vegetative and mid-reproductive developmental phase than on plants in the late-vegetative/early reproductive phase (Fig. 3b). 


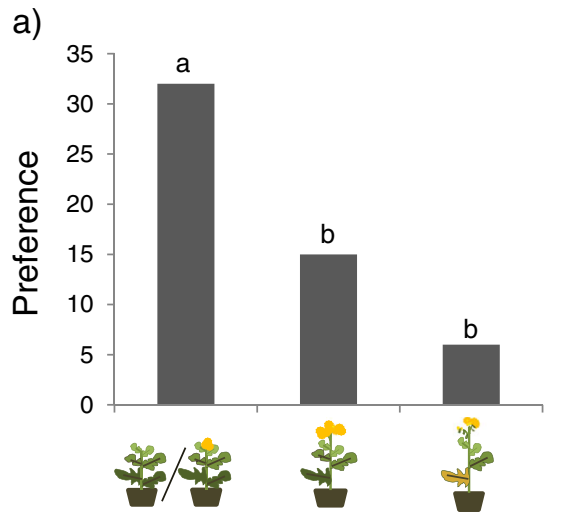

b)

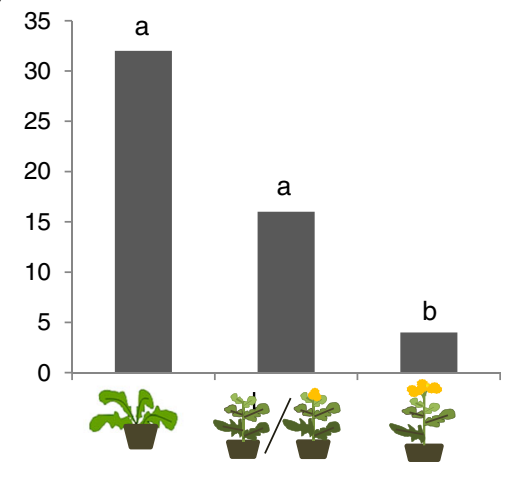

Developmental stage

Fig. 1 Counts of oviposition events by Pieris brassicae butterflies on Sinapis arvensis (a) and Brassica nigra (b) that were in different stages of development. $\rightarrow$ Represents vegetative phase, vegetative to early reproductive phase, phase, represents late-reproductive phase. Bars present the cumulative counts of oviposition choices ( $n=53$ for $S$. arvensis and $n=52$ for B. nigra), bars with the same letter are not significantly different (pairwise $\chi^{2}$-tests with a Bonferroni correction for multiple comparisons)

\section{Experiment 2b: Plant Ontogenetic Changes in Chemical} Profiles In leaf and flower tissues of S. arvensis 12 different glucosinolates, 7 sugars and 16 amino acids were detected, whereas in $B$. nigra 5 glucosinolates, 6 sugars and 16 amino acids were detected. In both plant species the differences in chemical profiles were most prominent between the two tissue types (Figs. 4 and 5). In B. nigra, differences between leaves and flowers primarily affected the quantity of individual compounds and not their quality (presence/absence). In addition to quantitative differences, some metabolites were only detected
Fig. 2 Effect of developmental stage on plant height $(\mathbf{a}, \mathbf{c})$ and total leaf surface area $(\mathbf{b}, \mathbf{d})$ in Sinapis arvensis $(\mathbf{a}, \mathbf{b})$ and Brassica nigra (c, d). A Represents vegetative phase,

\section{* * represents}

late-vegetative to

early-reproductive phase, represents mid-reproductive phase, represents late-reproductive phase. Bars present the means + SE (sample sizes are given in the bars) and bars with the same letter are not significantly different (Tukey-Kramer tests for multiple comparisons among means, $\alpha=0.05$ )
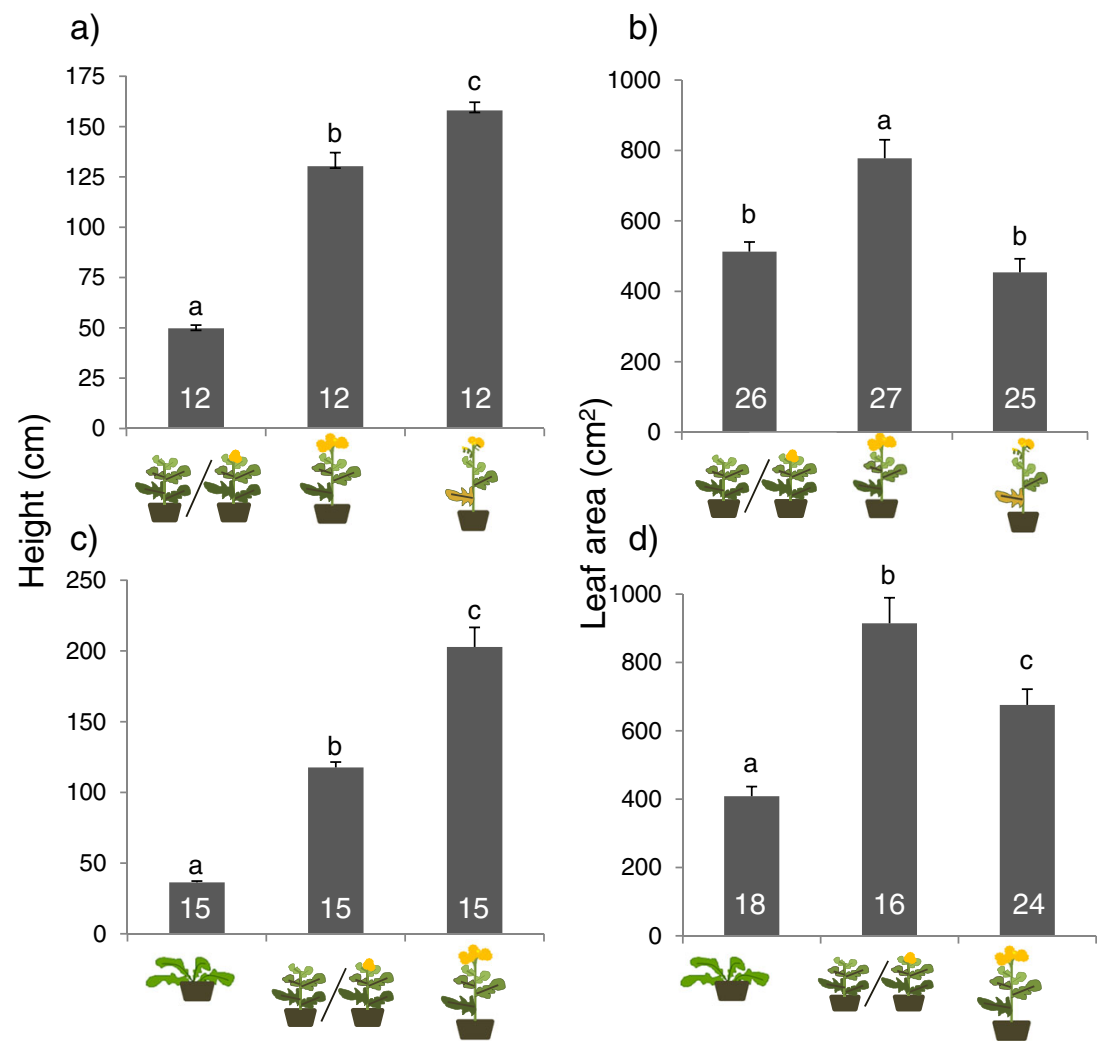

Developmental stage 
Fig. 3 Pupal fresh mass $(\mathbf{a}, \mathbf{b})$ and egg-to-pupa development time $(\mathbf{c}, \mathbf{d})$ of Pieris brassicae reared on three developmental stages of Sinapis arvensis $(\mathbf{a}, \mathbf{c})$ and Brassica nigra $(\mathbf{b}, \mathbf{d})$. $\mathrm{A}$ Represents vegetative phase, / represents late-vegetative and early-reproductive phase respectively, represents middle-reproductive phase, represents late-reproductive phase. Bars present the means $+\mathrm{SE}(n=5$ cages and $20 \mathrm{~L} 2$ caterpillars had been released in each cage) and bars with the same letter are not significantly different (Tukey-Kramer tests for multiple comparisons among means, $\alpha=0.05$ )

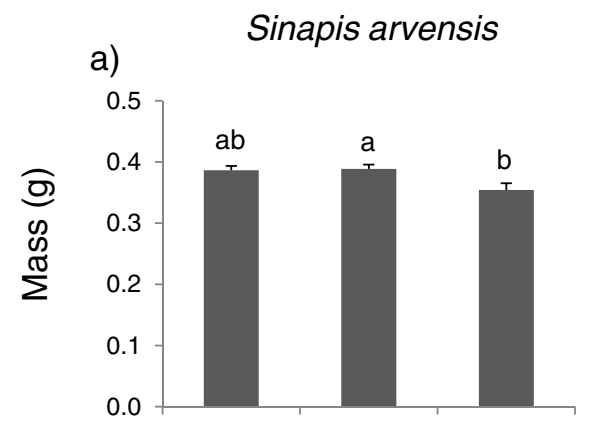

b) Brassica nigra

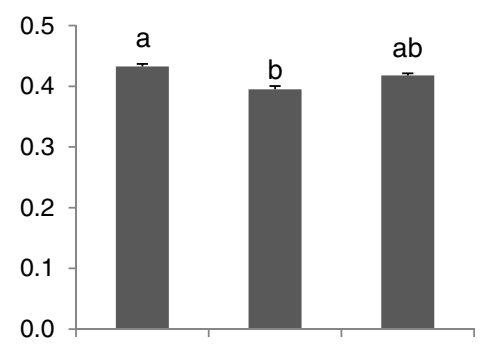

c)

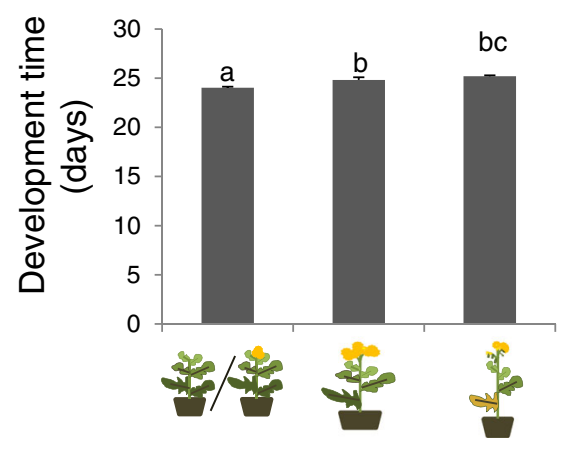

d)

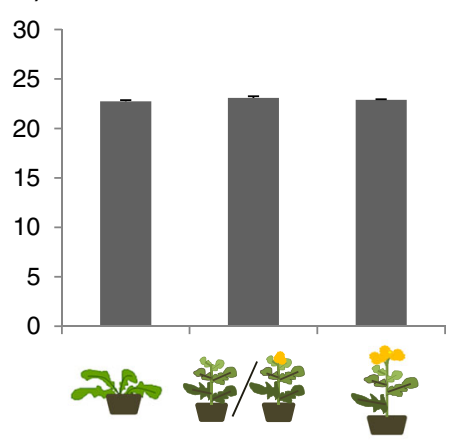

Developmental stage

in the flowers (leucine, tryptophan, 4-hydroxyglucobrassicin), and others (mannitol, melibiose, alanine) only in the leaves of S. arvensis. In both plant species, concentrations of many chemicals were higher in the flowers than in the leaves (Fig. 4), with the exception of the minor sugars, sorbitol, trehalose and melezitose, the amino acid, alanine and the indole glucosinolate 4-methoxyglucobrassicin, of which the concentrations were relatively higher in leaf tissues of both plant species (Fig. 5). Glucosinolate concentrations were also higher in the flowers than in the leaves, especially concentrations of the most dominant glucosinolates. In B. nigra, sinigrin (SIN), contributed $>98 \%$ to the total glucosinolate content in both the flowers and leaves, and in $S$. arvensis sinalbin (SNALB) contributed on average $60 \%$ and $90 \%$ to the glucosinolate content of the leaves and the flowers, respectively. Compared to differences related to tissue type, developmental stage and herbivory explained relatively little of the variation in chemistry attributes. Multivariate statistics confirmed this pattern (Table 1). For both plant species more than $80 \%$ of the variation in chemistry was attributed to differences between leaves and flowers and only a few percent was explained by plant developmental stage $(<4 \%)$ and whether plant tissues had been exposed to herbivory or not $(<2 \%)$.

Additional multivariate statistical models analyzing flower and leaf data separately with only developmental stage and herbivory status as explanatory variables revealed that these factors affected chemical profiles of the leaves stronger than those of the flowers, as the variation explained by these factors was higher for the leaves than for the flowers (Table 1, the graphs are given in the Supplementary Material). Chemical profiles of the flowers of $B$. nigra could not be separated according to developmental stage or herbivory status. In B. nigra, younger leaf tissues tended to have higher levels of most amino acids and the sugars, sorbitol and glucose, whereas levels of trehalose and alanine tended to be higher in the leaves of young and middleaged $B$. nigra plants. Levels of the amino acids glutamine, tyrosine and tryptophan were relatively higher in damaged than in control B. nigra leaf tissues. The effects of developmental stage and damage status on glucosinolate concentrations were relatively small in this plant species. Contrastingly, in the leaves of $S$. arvensis, sinalbin, the dominant glucosinolate in this plant species, decreased with plant age, whereas in the flowers the opposite pattern was found for this compound. Concentrations of sinalbin and the indole glucosinolate 4-methoxyglucobrassicin were also higher in damaged than in control tissues. Concentrations of most sugars increased with age of the plants, both in the flowers and the leaves. The changes in amino acid concentrations in relation to plant age were more idiosyncratic. Sorbitol glutamine, and aspartic acid were relatively high in damaged leaves, whereas melibiose, sucrose and isoleucine were relatively high in undamaged control leaf tissues. Tryptophan was relatively high in control tissues and sinalbin was relatively high in damaged tissues.

Linking Insect Performance to Plant Chemistry For S. arvensis, significant correlations were found between insect 
Fig. 4 Total concentrations (mean $+\mathrm{SE})$ of glucosinolates (a, b) amino acids (c, d) and sugars $(\mathbf{e}, \mathbf{f})$ in leaf and flower tissues of Sinapis arvensis $(\mathbf{a}, \mathbf{c}, \mathbf{e})$ and Brassica nigra (b, d, f) that were sampled from plants in three different developmental stages. 24. Represents vegetative phase, * to early reproductive phase, represents mid-reproductive phase, represents 1 ate-reproductive phase. In each panel metabolite concentrations in $\mu \mathrm{mol} \mathrm{g}{ }^{-1}$ dry weight (DW) are given for the leaves (left) and flowers (right) and for undamaged control plants (=ct) and plants exposed to Pieris brassicae feeding (=dam). Colours within bars refer to different compounds or classes of compounds (glucosinolates). Each bar represents the mean + SE (5 biological replicates) of pooled samples of 5 discs from each of 4 leaves or 8 flowers collected from two plants per replicate
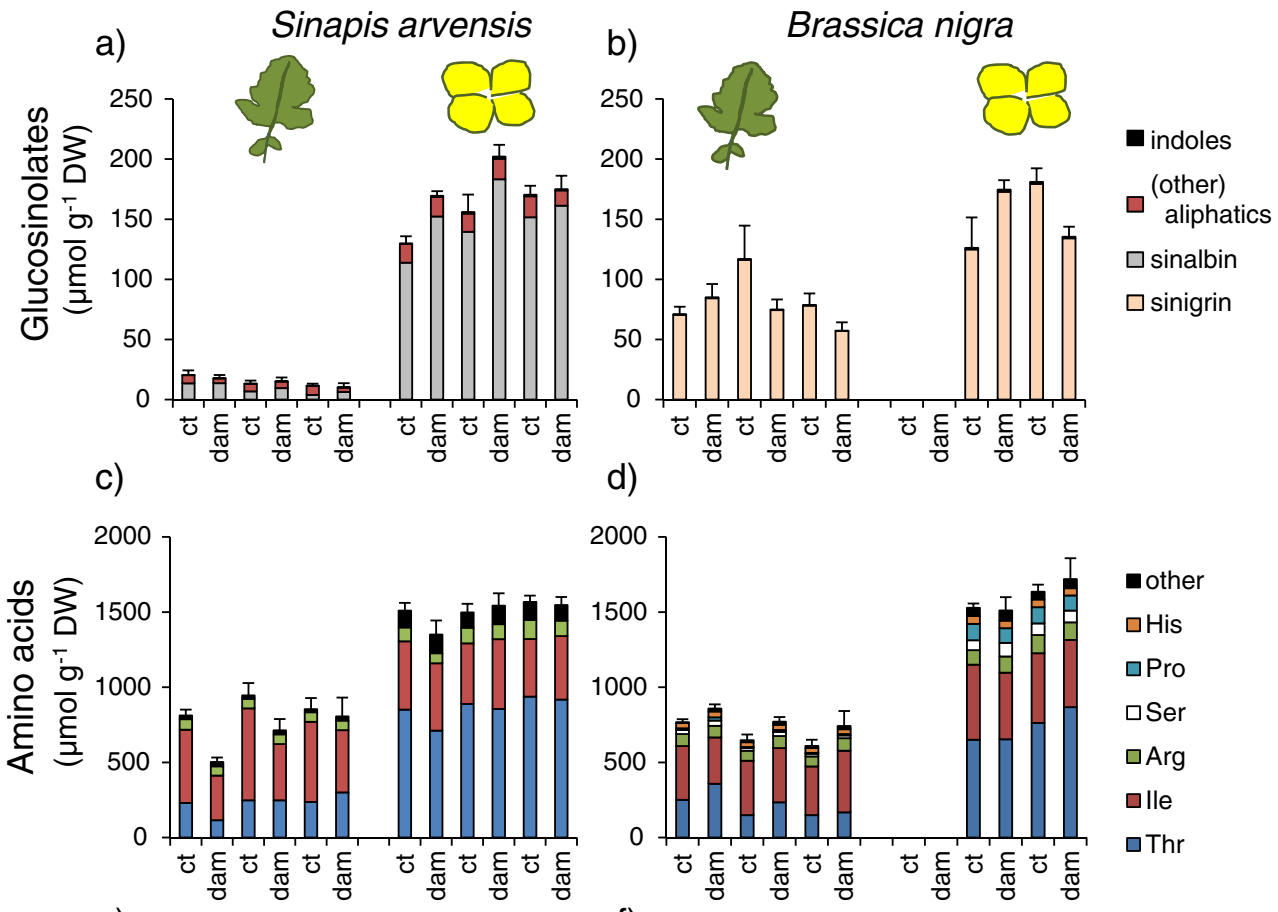

d)
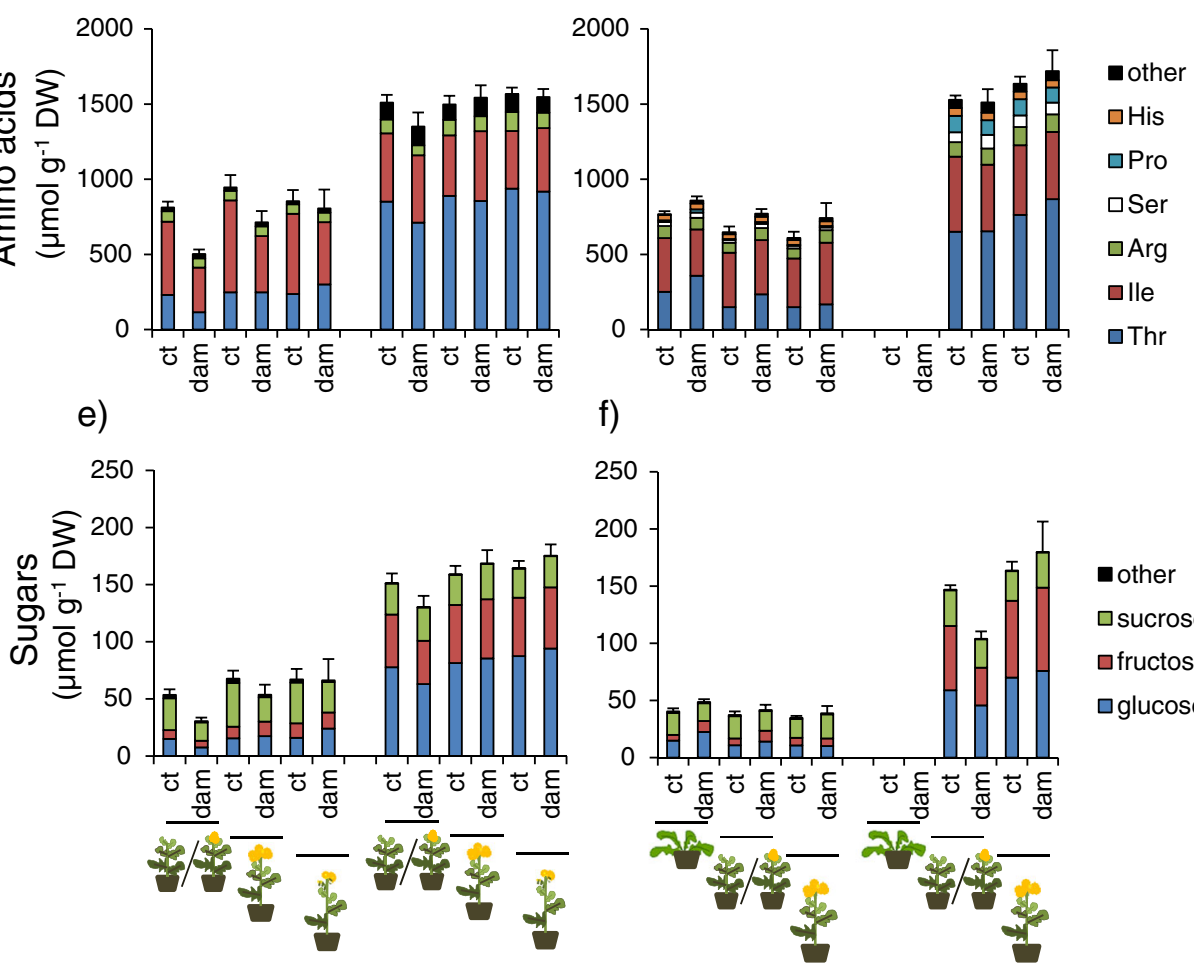

f)

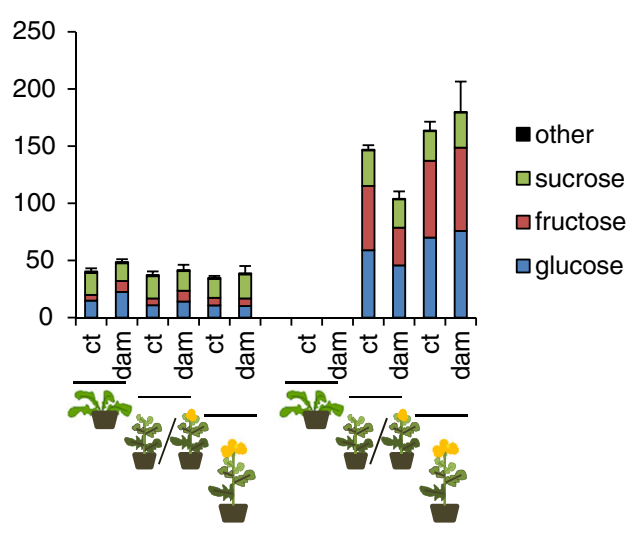

Plant developmental stage

performance variables and plant chemistry for both the leaves (RDA, $P=0.004$ ) and the flowers (RDA, $P=0.048$ ). For $B$. nigra, significant correlations were found between insect performance variables and plant chemistry for the flowers (RDA, $P=0.034$ ), but not for the leaves (RDA, $P=0.70$ ). However, no consistency in chemical profiles explaining insect performance could be detected when comparing the two plant species or intra-specifically comparing leaves and flowers.

\section{Discussion}

When female $P$. brassicae butterflies were presented with different developmental stages of $S$. arvensis or B. nigra plants, acceptance for oviposition decreased with plant age. Interestingly, selection of plants negatively correlated with plant size, i.e. the smallest plants were preferred for oviposition. On S. arvensis plants varying in age, oviposition preference of adult females correlated weakly with both larval development time and pupal mass. Caterpillars developed fastest and attained the largest biomass as pupae when they grew on the youngest plants, i.e. plants in the vegetative /early reproductive stage. No such correlation was found for B. nigra. Caterpillars developed into heavier pupae on $B$. nigra plants in the vegetative and mid-reproductive stage and were lighter on plants in the early reproductive stage, whereas there was no effect on development time. Chemical analyses of glucosinolates, amino acids and sugars in leaf and flower tissues collected from the two plant species in different developmental stages revealed that the differences in the analyzed plant metabolites were more evident with respect to tissue type than among different developmental stages or whether plants were 
a)

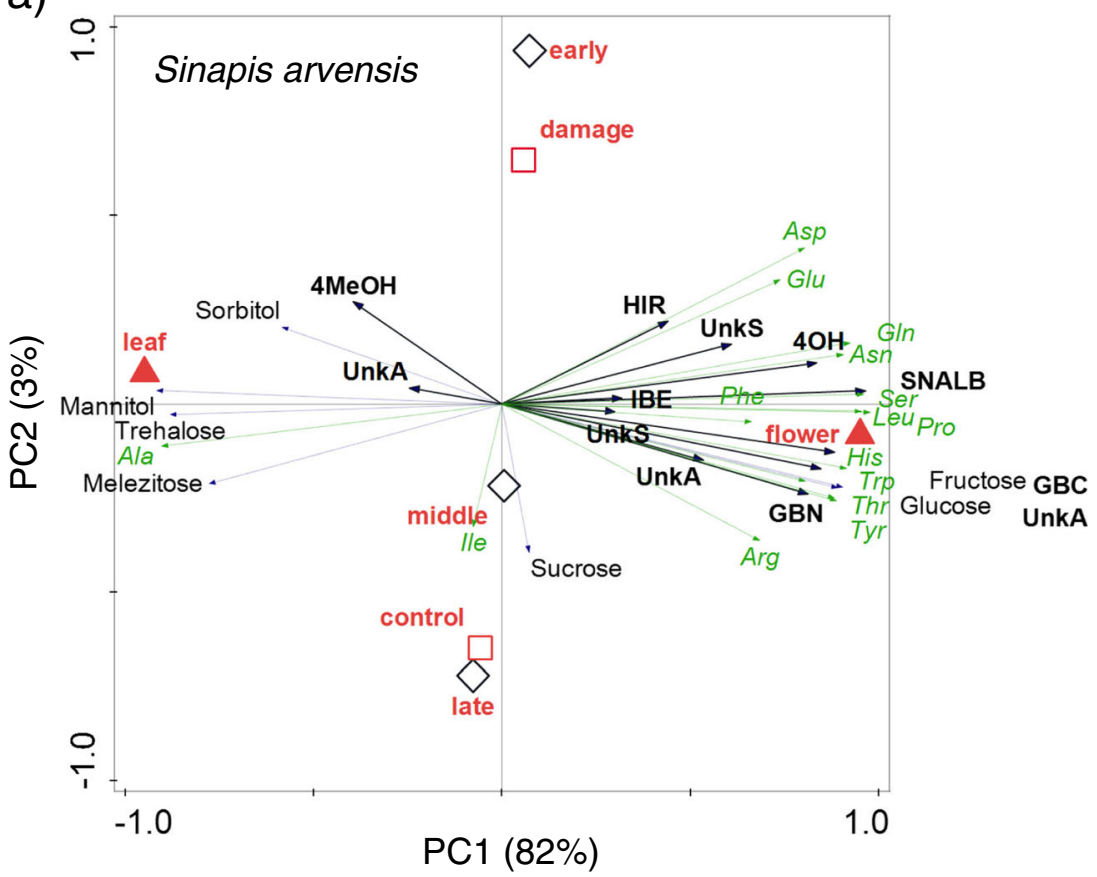

b)

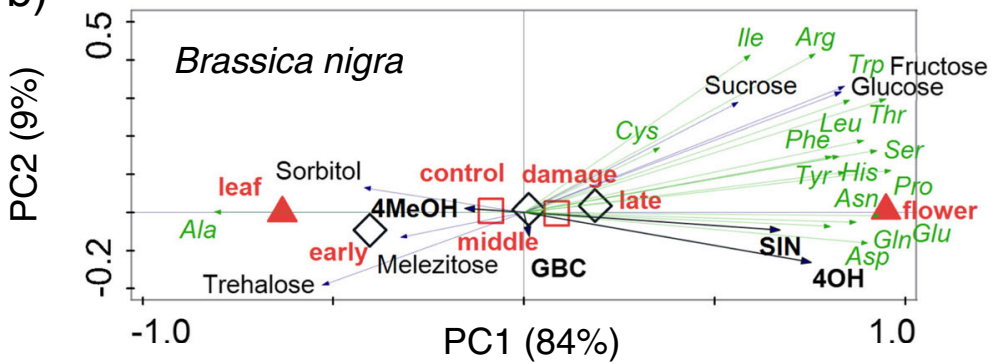

Fig. 5 Redundancy analyses (RDA) on the chemical profiles (sugar, amino acid and glucosinolate (GS) concentrations) of Sinapis arvensis (a) and Brassica nigra (b) leaf and flower tissues. The statistics are given in Table 1. The centroids depict mean scores for the various classes; leaf and flower tissues (red triangles), plant developmental stages (black open diamonds) and herbivore-damaged or control tissues (red open squares). The loadings (contribution of the chemical variables) are given in solid black lines and arrows for the GS, dotted green lines and arrows for the amino acids, and black dotted lines for the sugars. The distance from the axes origin to each of the arrows indicates the magnitude of the contribution of the variable to the principal components. For the amino acids (green) the international IUPAC three letter codes were used. Sugar names are in black and glucosinolate names are in bold black font where

damaged or not. Although for both plants species correlations were found between insect performance and plant chemistry variables, we did not detect clear consistencies in phytochemistry characteristics and insect performance.

Host plant selection for ovipostion sites by adult insect herbivores is a complex behavioral process that is guided by various sensory inputs (Carrasco et al. 2015; Finch and Collier 2000). Female butterflies select host plants for oviposition based on visual, olfactory and gustatory information (Bruce et al. 2005; Schoonhoven et al. 2005). In some species,
SIN = sinigrin (2-propenyl GS), GBN = glucobrassicanapin (3-butenyl GS), $\mathrm{IBE}=$ glucoiberin (3-methylsulfinylpropyl GS), $\mathrm{HIR}=$ glucohirsutin (8-methylsulfinyloctyl GS =, UnkA = unknown aliphatic GS (not further specified), UnkS = unknown sulfur containing GS, GBC = glucobrassicin (indol-3-ylmethyl GS), 4OH = 4-hydroxyglucobrassicin (4-hydroxyindol-3-ylmethyl GS), 4MeOH = 4-methoxyglucobrassicin (4-methoxyindol-3-ylmethyl GS), SNALB = sinalbin (4hydroxbenzyl GS). In 5a, early represents late-vegetative/early reproductive phase, middle represents mid-reproductive phase, and late represents late-reproductive phase. In 5b, early represents early-vegetative phase, middle represents late-vegetative/early reproductive phase, and late represents middle-reproductive phase

concentrations or proportions of particular chemical compounds determine oviposition preference of female butterflies (Thompson and Pellmyr 1991). Caterpillars of P. brassicae are specialist feeders on plants containing glucosinolates, mainly species in the family Brassicaceae (Feltwell 1982). Studies have shown that glucosinolates stimulate oviposition by adult females and feeding by the larvae, respectively, although not all glucosinolate compounds do this to the same extent (Renwick et al. 1992; van Loon et al. 1992). Previous studies have shown that host plant recognition for oviposition 
Table 1 Multivariate RDA statistics using chemical data of flowers and leaves together or separately

\begin{tabular}{|c|c|c|c|c|c|c|c|c|c|c|}
\hline \multirow[t]{2}{*}{ Factors } & \multicolumn{3}{|c|}{ Tissue type } & \multicolumn{3}{|c|}{ Developmental stage } & \multicolumn{3}{|c|}{ Herbivory (yes/no) } & \multirow[b]{2}{*}{$\mathrm{PCs}^{\mathrm{C}}$} \\
\hline & $\mathrm{F}^{\mathrm{a}}$ & $P^{\mathrm{b}}$ & $\% \operatorname{expl}^{\mathrm{c}}$ & $\mathrm{F}$ & $P$ & $\%$ expl. & $\mathrm{F}$ & $P$ & $\%$ expl & \\
\hline \multicolumn{11}{|c|}{ Plant species } \\
\hline S. arvensis & 312 & $\leq 0.001$ & 85.5 & 4.4 & $\leq 0.001$ & 1.9 & 7.3 & $\leq 0.001$ & 1.7 & 3 \\
\hline Flowers & & & & 3.3 & $\leq 0.001$ & 13.2 & 4.1 & 0.003 & 9.0 & 2 \\
\hline Leaves & & & & 5.4 & $\leq 0.001$ & 20.1 & 8.6 & $\leq 0.001$ & 17.9 & 2 \\
\hline B. nigra & 233 & $\leq 0.001$ & 81.6 & 5.6 & $\leq 0.001$ & 3.1 & 3.7 & 0.02 & 0.9 & 1 \\
\hline Flowers & & & & 1.0 & 0.40 & 0.2 & 0.8 & 0.64 & 0 & 0 \\
\hline Leaves & & & & 9.5 & $\leq 0.001$ & 28.4 & 17.0 & $\leq 0.001$ & 27.7 & 2 \\
\hline
\end{tabular}

${ }^{\text {a }}$ Pseudo $\mathrm{H}_{0}$ F-statistic used in the Monte Carlo permutation test

${ }^{\mathrm{b}}$ Significance level of the Monte Carlo permutation test

${ }^{\mathrm{c}}$ Adjusted percentage of the explained variation

${ }^{\mathrm{d}} \mathrm{PCs}$ are the number of significant principal components $(P<0.05)$ in each model

by $P$. brassicae butterflies requires physical contact with the plant and is based on chemoreception of glucosinolates on the leaf surface (Renwick 2002; van Loon et al. 1992). On the leaves, where $P$. brassicae females habitually lay their eggs, concentrations of the dominant glucosinolate only decreased with plant age in S. arvensis and not in B. nigra. Van Loon et al. (1992) found that glucobrassicin is a much stronger oviposition stimulant than sinigrin, the dominant glucosinolate in B. nigra. The concentration of glucobrassicin was found to be very low in undamaged foliage of $B$. nigra and S. arvensis $\left(<0.8\right.$ and $<0.06 \mu \mathrm{mol} \mathrm{g}^{-1} \mathrm{DW}$, respectively) and it did not change with plant development. Thus, sinigrin and glucobrassicin concentrations do not explain oviposition preference in relation to plant age in this study. Detection of glucosinolates by $P$. brassicae butterflies occurs most likely at the surface of the leaf. We do not know how whole-leaf glucosinolate content relates to that at the leaf surface.

Females of $P$. brassicae typically lay eggs in clutches varying between 10 and 50, although more than 100 eggs may be laid during one oviposition event. Depending on the number of caterpillars and the size of the food plants, several plants growing in close proximity are needed for the successful development of $P$. brassicae larvae from a single egg clutch. Thus both quantitative and qualitative characteristics of the food plant determine development and survival of P. brassicae caterpillars (Fei et al. 2016a). One area that is rarely considered in studies of oviposition behavior and plant preference in herbivores, however, is that the performance of offspring is not necessarily determined by current plant quality and quantity but by the ways in which these parameters are affected in the future. The plant is not a static resource during insect development, but is also growing and developing itself. Depending on local abiotic conditions like temperature, the entire egg-to-pupa development period may cover a month or more. Furthermore, as in most holometabolous insects, >
$80 \%$ of insect feeding and growth in P. brassicae occurs during the final (= L5) instar. This stage can occur some 34 weeks after oviposition, meaning that the mother may have to be able to roughly predict plant quality (and quantity) several weeks in advance when selecting a plant on which to oviposit. Given that their short-lived food plants may be present for only 2-3 months in the field, this represents a significant fraction of the plant's lifetime. P. brassicae may select plants not on the basis of present quality or quantity but more importantly on anticipating future quality and quantity coinciding with the final instar of their larvae. Laying eggs on plants in the mid to late reproductive phase may have serious consequences for offspring fitness and survival as plant tissues may be senescing by the time the larvae reach their final instar. Female butterflies may determine growth potential of fast growing annual plants based on chemical, visual and tactile sensory input or a combination of these.

Ontogenetic variation in primary and secondary metabolites may also influence the performance of insects feeding on plants differing in age (Quintero et al. 2014). In general, insects tend to prefer, have higher densities, and develop better on younger than on older plants (Boege and Marquis 2005). The developmental stage of the plants in this study had relatively small effects on P. brassicae larval performance variables such as development time and pupal mass and survival to pupation. Comparing chemical profiles of leaf and flower tissues collected from plants in different developmental stages revealed that differences in plant chemistry were more pronounced with respect to tissue type than to developmental stage. In addition to ontogenetic changes in chemistry, concentrations of plant phytochemicals also change in response to herbivory (Karban and Baldwin 1997) and herbivore-induced plant responses may also depend on plant ontogeny (Barton and Koricheva 2010; Quintero and Bowers 2011; van Dam 
et al. 2001). No consistencies were found for the two plant species in plant age-related and herbivore-induced changes in metabolite concentrations. Moreover, correlation analysis of insect performance and plant chemistry also did not reveal consistent patterns explaining insect performance in relation to plant chemistry. We only measured a selected number of chemical variables in the plant. Plant quality as food for caterpillars is not restricted to the compounds analyzed in this study. Phosphorous, lipids, ratios of certain amino acids / proteins, digestibility reducing enzymes, cellulose content, and the presence of other plant secondary metabolites may affect digestibility of the ingested food and contribute to the overall nutritional quality of the food plant (Speight et al. 2008). However, the results of this study suggest that if other plant nutritional traits varied in relation to plant age, their effects on larval performance were limited, especially in $B$. nigra.

Whereas young larvae of $P$. brassicae feed only on leaf tissues, older caterpillars move up the plant and prefer to feed on the flowers when present (Smallegange et al. 2007). Moreover, caterpillars that only had access to flowers developed faster and attained a greater final larval mass than conspecifics that only had access to $B$. nigra leaves (Smallegange et al. 2007). In our experiments, we allowed the caterpillars to move and feed freely on the leaves and the flowers. The mixed diet of the caterpillars may further explain the poor correlation between phytochemistry and insect performance. Nevertheless, it is notable that despite the large differences in chemistry between the leaves and the flowers, pupal mass and larval development time were relatively little affected. For example, the performance of caterpillars was similar on young $B$. nigra plants that had not yet produced flowers, and old $B$. nigra plants that had developed flowers. The discrepancy between the results reported in Smallegange et al. (2007) and this study can also be explained by the fact that larval mass (Smallegange et al. 2007) and pupal mass (this study) are not necessarily positively correlated (Ansari et al. 2012). Moreover, in the study by Smallegange et al. (2007) a single age class of $B$. nigra plant was used. Pieris brassicae has evolved to feed on a range of short lived annual species that all contain glucosinolates but also exhibit considerable variation in their chemical profiles, both quantitatively and qualitatively. The variation in plant quality among the plants used in this study may have been too small to strongly impact on larval performance (see also Gripenberg et al. 2010).

One critical point that is often ignored when studying behavior is that preference may be reduced in insect colonies that have been reared under standardized conditions in the laboratory, especially with respect to food quality. However, we found that female butterflies were still 'choosy' with respect to oviposition decisions. Cultures of our butterflies are partially replenished every summer, thus mitigating this effect. Moreover, in a recent study, we also found that oviposition preference of adult $P$. brassicae butterflies changed when they had developed as caterpillars on different host plant species (Fei et al. 2016b). Interestingly, these butterflies never preferred to oviposit on the plant species on which they had developed as caterpillars (Fei et al. 2016b). In combination, these results reveal that $P$. brassicae retains selectivity even when reared in the laboratory.

According to the 'preference-performance' hypothesis, insect herbivores should lay their eggs on host plants that are most suitable for development and survival of their offspring (Jaenike 1978). However, the results of studies on the relationship between adult oviposition preference and larval performance do not always corroborate this hypothesis (Gripenberg et al. 2010; Jaenike 1990; Mayhew 2001; Thompson and Pellmyr 1991;). Fitness benefits associated with host plants may differ for the adult mothers and her offspring (Scheirs et al. 2005). Oviposition preference and larval performance may be poorly correlated when the larval host plant is bad for the mother (e.g. when physical characteristics hamper egg laying, but not larval feeding), when there is variability in host-plant availability and quality in time and space, and when food plants are chosen by the offspring (Mayhew 2001; Thompson 1988; Wiklund and Friberg 2009). Other ecological factors, such as reduced risk of attack by the herbivore's natural enemies, or enemy free space (Mira and Bernays 2002; Oppenheim and Gould 2002) and interactions with mutualists preventing predation (Atsatt 1981) may also play important roles when selecting plants for oviposition. Moreover, female physiology (age, egg loads) and optimization of survival of the eggs rather than the larvae (Tammaru et al. 1995) may further explain why adult preference and offspring performance are not necessarily positively correlated. Both $B$. nigra and $S$. arvensis have also been reported to respond to $P$. brassicae egg deposition by forming necrotic tissues, which negatively impacts survival of the eggs and growth and development of the caterpillars developing from these eggs (Hilker and Fatouros 2015; Pashalidou et al. 2015). Generally, inducibility of plant responses to herbivory tends to decrease with plant age (Barton and Koricheva 2010). In B. nigra, the response to egg deposition was also reduced in older plants (D. Lucas-Barbosa, unpublished data), but this did not appear to affect oviposition preference of $P$. brassicae in this study. As an alternative strategy, we propose that oviposition decisions may be based on future and not present plant quality. The biology of the plant, in terms of the rate of growth and dynamic changes in primary and secondary chemistry may also depend on life-history traits of the plant, e.g., whether the plant is a long-lived perennial or a short-lived annual. Thus preference-performance studies need to account for differences in the biology of the food plants and the biology of the herbivore.

In summary, our study reveals that adult $P$. brassicae female butterflies distinguish between different 
developmental stages of their food plants and prefer younger stages of both $S$. arvensis and B. nigra for oviposition, whereas the consequences of these preferences for larval growth and development were relatively small. In the Netherlands $S$. arvensis and B. nigra, which often grow in large stands, are important food plant species for $P$. brassicae. For a multivoltine insect herbivore like $P$. brassicae that primarily feeds on short-lived annuals, it is a challenge for different generations to locate suitable host plants. Preference for younger host plants may allow the plant to grow additional resources to support growth of the larvae that feed gregariously and are particularly voracious during the final instar. For a multivoltine insect that feeds on short-lived, fast growing plants it is also adaptive to be able to exploit a broad range of food plants and to be little affected by ontogenetic changes in plant quality.

Acknowledgements Ciska Raaijmakers is thanked for helping with the chemical analyses. This work is supported by a grant from the China Scholarship Council.

\section{Compliance with Ethical Standards}

Conflict of Interest The authors declare that they have no conflict of interest.

Ethical Approval All applicable institutional and/or national guidelines for the care and use of animals were followed.

Open Access This article is distributed under the terms of the Creative Commons Attribution 4.0 International License (http:// creativecommons.org/licenses/by/4.0/), which permits unrestricted use, distribution, and reproduction in any medium, provided you give appropriate credit to the original author(s) and the source, provide a link to the Creative Commons license, and indicate if changes were made.

\section{References}

Ansari MS, Hasan F, Ahmad N (2012) Influence of various host plants on the consumption and utilization of food by Pieris brassicae (Linn.) Bull Entomol Res 102:231-237. doi:10.1017/S000748531100054x

van Asch M, Julkunen-Tiito R, Visser ME (2010) Maternal effects in an insect herbivore as a mechanism to adapt to host plant phenology. Funct Ecol 24:1103-1109

Atsatt PR (1981) Ant-dependent food plant-selection by the mistletoe butterfly Ogyris amaryllis (Lycaenidae). Oecologia 48:60-63. doi: 10.1007/Bf00346988

Barton KE, Koricheva J (2010) The ontogeny of plant defense and herbivory: characterizing general patterns using meta-analysis. Am Nat 175:481-493. doi:10.1086/650722

Boege K, Marquis RJ (2005) Facing herbivory as you grow up: the ontogeny of resistance in plants. Trends Ecol Evol 20:441-448

Bowers MD, Stamp NE (1993) Effects of plant age, genotype and herbivory on Plantago performance and chemistry. Ecology:17781791 ter Braak CJF, Šmilauer P (2012) Canoco reference manual and User's guide, software for Odination (version 5.0). Microcomputer power, Ithaca

Bruce TJA, Wadhams LJ, Woodcock CM (2005) Insect host location: a volatile situation. Trends Plant Sci 10:269-274

Carrasco D, Larsson MC, Anderson P (2015) Insect host plant selection in complex environments. Curr Opin Insect Sci 8:1-7

Cronin JT, Abrahamson WG, Craig TP (2001) Temporal variation in herbivore host-plant preference and performance: constraints on host-plant adaptation. Oikos 93:312-320. doi:10.1034/j.16000706.2001.930214.x

van Dam NM, Oomen MWAT (2008) Root and shoot jasmonic acid applications differentially affect leaf chemistry and herbivore growth. Plant Signal Behav 3:91-98

van Dam NM, Horn M, Mares M, Baldwin IT (2001) Ontogeny constrains systemic protease inhibitor response in Nicotiana attenuata. $\mathrm{J}$ Chem Ecol 27:547-568

van Dam NM, Witjes L, Svatos A (2004) Interactions between aboveground and belowground induction of glucosinolates in two wild Brassica species. New Phytol 161:801-810

Despres L, David JP, Gallet C (2007) The evolutionary ecology of insect resistance to plant chemicals. Trends Ecol Evol 22:298-307

Fahey JW, Zalcmann AT, Talalay P (2001) The chemical diversity and distribution of glucosinolates and isothiocyanates among plants. Phytochemistry 56:5-51

Fei MH, Gols R, Harvey JA (2014) Seasonal phenology of interactions involving short-lived annual plants, a multivoltine herbivore and its endoparasitoid wasp. J Anim Ecol 83:234-244

Fei M, Gols R, Zhu F, Harvey JA (2016a) Plant quantity affects development and survival of a gregarious insect herbivore and its endoparasitoid wasp. PLoS One :e0149539. doi:10.1371/journal. pone.0149539

Fei MH, Harvey JA, Weldegergis BT, Huang T, Reijngoudt K, Vet LM, Gols R (2016b) Integrating insect life history and food plant phenology: flexible maternal choice is adaptive. Int J Mol Sci 17:e1263. doi:10.3390//jms17081263

Feltwell J (1982) Large white butterfly, the biology, biochemistry and physiology of Pieris brassicae (Linaeus). Dr W. Junk Publishers, The Hague

Finch S, Collier RH (2000) Host-plant selection by insects - a theory based on 'appropriate/inappropriate landings' by pest insects of cruciferous plants. Entomol Exp Appl 96:91-102

Fraenkel GS (1959) Raison d'être of secondary plant substances. Science 129:1466-1470

Gols R, Raaijmakers CE, van Dam NM, Dicke M, Bukovinszky T, Harvey JA (2007) Temporal changes affect plant chemistry and tritrophic interactions. Basic Appl Ecol 8:421-433

Gripenberg S, Mayhew PJ, Parnell M, Roslin T (2010) A meta-analysis of preference-performance relationships in phytophagous insects. Ecol Lett 13:383-393

Hilker M, Fatouros NE (2015) Plant responses to insect egg deposition. Annu rev Entomol 60:493-515. doi:10.1146/annurev-ento-010814020620

Iason GR, Dicke M, Hartley SE (2012) The ecology of plant secondary metabolites : from genes to global processes. Cambridge University Press, Cambridge, Ecological reviews

Jaenike J (1978) On optimal oviposition behavior in phytophagous insects. Theor Popul Biol 14:350-356

Jaenike J (1990) Host specialization in phytophagous insects. Annu Rev Ecol Syst 21:243-273

Karban R, Baldwin IT (1997) Induced responses to herbivory. University of Chicago Press, Chicago

Lawton J, McNeill S (1979) Between the devil and the deep blue sea: on the problem of being a herbivore. Symp Brit Ecol Soc 20:223-244

van Loon JJA, Blaakmeer A, Griepink FC, van Beek TA, Schoonhoven LM, de Groot A (1992) Leaf surface compound from Brassica 
oleracea (Cruciferae) induces oviposition by Pieris brassicae (Lepidoptera: Pieridae). Chemoecology 3:39-44

Loxdale HD, Lushai G, Harvey JA (2011) The evolutionary improbability of 'generalism' in nature, with special reference to insects. Biol J Linn Soc 103:1-18. doi:10.1111/j.1095-8312.2011.01627.x

Mattson WJ (1980) Herbivory in relation to plant nitrogen-content. Annu Rev Ecol Syst 11:119-161

Mayhew PJ (1998) Testing the preference-performance hypothesis in phytophagous insects: lessons from chrysanthemum leafminer (Diptera : Agromyzidae). Environ Entomol 27:45-52

Mayhew PJ (2001) Herbivore host choice and optimal bad motherhood. Trends Ecol Evol 16:165-167. doi:10.1016/S0169-5347(00)02099-1

Mira A, Bernays EA (2002) Trade-offs in host use by Manduca sexta: plant characters vs natural enemies. Oikos 97:387-397. doi:10. 1034/j.1600-0706.2002.970309.x

Mithöfer A, Boland W (2012) Plant defense against herbivores: chemical aspects. Ann Rev Plant Biol 63:431-450

Nishida R (2002) Sequestration of defensive substances from plants by Lepidoptera. Annu Rev Entomol 47:57-92

Oppenheim SJ, Gould F (2002) Behavioral adaptations increase the value of enemy-free space for Heliothis subflexa, a specialist herbivore. Evolution 56:679-689

Pashalidou FG, Fatouros NE, Van Loon JJA, Dicke M, Gols R (2015) Plant-mediated effects of butterfly egg deposition on subsequent caterpillar and pupal development, across different species of wild Brassicaceae. Ecol Entomol 40:444 450. doi:10.1111/een.12208

Quintero C, Bowers MD (2011) Plant induced defenses depend more on plant age than previous history of damage: implications for plantherbivore interactions. J Chem Ecol 37:992-1001

Quintero C, Lampert EC, Bowers MD (2014) Time is of the essence: direct and indirect effects of plant ontogenetic trajectories on higher trophic levels. Ecology 95:2589-2602

Renwick J (1989) Chemical ecology of oviposition in phytophagous insects. Experientia 45:223-228

Renwick JAA (2002) The chemical world of crucivores: lures, treats and traps. Entomol Exp Appl 104:35-42
Renwick JAA, Radke CD, Sachdev-Gupta K, Städler E (1992) Leaf surface chemicals stimulating oviposition by Pieris rapae (Lepidoptera: Pieridae) on cabbage. Chemoecology 3:33-38

Scheirs J, Jordaens K, De Bruyn L (2005) Have genetic trade-offs in host use been overlooked in arthropods? Evol Ecol 19:551-561. doi:10. 1007/s10682-005-2004-y

Schoonhoven LM, van Loon JJA, Dicke M (2005) Insect-plant biology, 2nd edn. Oxford University Press, Oxford

Scriber JM, Slansky F (1981) The nutritional ecology of immature insects. Annu Rev Entomol 26:183-211

Smallegange RC, van Loon JJA, Blatt SE, Harvey JA, Agerbirk N, Dicke M (2007) Flower $v s$. leaf feeding by Pieris brassicae: glucosinolaterich flower tissues are preferred and sustain higher growth rate. $\mathrm{J}$ Chem Ecol 33:1831-1844

Speight MR, Hunter MD, Watt AD (2008) Ecology of insects: concepts and applications. Wiley-Blackwell, Oxford

Tammaru T, Kaitaniemi P, Ruohomäki, K (1995) Oviposition choices of Epirrita autumnata (Lepidoptera: Geometridae) in relation to its eruptive population dynamics. Oikos 74:296-304. doi:10.2307/ 3545659

Thompson JN (1988) Evolutionary ecology of the relationship between oviposition preference and performance of offspring in phytophagous insects. Entomol Exp Appl 47:3-14

Thompson JN, Pellmyr O (1991) Evolution of oviposition behavior and host preference in Lepidoptera. Annu Rev Entomol 36:65-89

Wiklund C, Friberg M (2009) The evolutionary ecology of generalization: among-year variation in host plant use and offspring survival in a butterfly. Ecology 90:3406-3417. doi:10. 1890/08-1138.1

Winde I, Wittstock U (2011) Insect herbivore counteradaptations to the plant glucosinolate-myrosinase system. Phytochemistry 72:15661575. doi:10.1016/j.phytochem.2011.01.016

Wolda H (1988) Insect seasonality - why? Annu Rev Ecol Syst 19:1-18 